\title{
Influence of government-driven quality assessment program on patients with chronic obstructive pulmonary disease
}

\author{
Hye Jung Park', Sung-Ryeol Kim²,3, Sinae Kim, Hye Sun Lee ${ }^{5}$, Bo Yeon Kim ${ }^{6}$, Hye Kyoung Kim ${ }^{6}$, Sang In Ahn',
} Ji Hyeon Shin', Jae-Hyun Lee ${ }^{2,3^{*}}$ (10 and Jung-Won Park ${ }^{2,3}$

\begin{abstract}
Background: The Korean Health Insurance Review and Assessment Service (HIRA) has launched the Chronic Obstructive Pulmonary Disease (COPD) Quality Assessment Program (CQAP) since 2014. We aimed to reveal the influence of this national program on clinical outcomes and the burden of COPD in Korea.

Methods: The CQAP is conducted annually. We used healthcare claims data linked with the results of the program provided by HIRA between May 2014 and April 2017. Patients were considered to have COPD if they visited a hospital for COPD management during the assessment term. Those who visited a medical institution for COPD and were prescribed COPD medications at least twice were assessed by the CQAP (assessed subjects, AS; not-assessed subjects, NAS). CQAP evaluated the pulmonary function test conduction rate, regular visitation rate, and prescription rates of COPD medications.

Results: Among the 560,000 patients with COPD, about 140,000 were assessed by the CQAP annually. In both groups, the pulmonary function test conduction rate and inhaled bronchodilator prescription rate improved since 2014. Compared to the NAS group, the risk of admission and all-cause mortality rate in the AS group were significantly reduced by $21.2 \%$ and $40.7 \%$, respectively. In patients who were assessed for 3 consecutive years, all of the above variables were high at baseline and were not improved much from implementation of CQAP. In matching analysis, we observed this improvement to be limited in the COPD quality assessment year.
\end{abstract}

Conclusions: The CQAP by the health insurance bureau has improved the management protocol and prognosis of COPD.

Keywords: Chronic obstructive pulmonary disease, Quality assessment, Prognosis, Clinical outcome, Mortality rate

\section{Introduction}

Chronic obstructive pulmonary disease (COPD) is a chronic airway inflammatory disease characterized by a progressive decline in lung function $[1,2]$. Therefore, affected patients require close monitoring of symptoms and pulmonary function and engagement in active

\footnotetext{
*Correspondence: jhleemd@yuhs.ac

2 Division of Allergy and Immunology, Department of Internal Medicine, Yonsei University College of Medicine, Seoul, Republic of Korea

Full list of author information is available at the end of the article
}

medical treatment with bronchodilator-based regimen [3]. The worldwide prevalence of COPD is $10.1 \%$ with the number of patients continuously increasing, and it also increase the socioeconomic burden $[4,5]$. Pulmonary function tests (PFTs) are essential for the diagnosis and follow-up of COPD, and a low $\mathrm{FEV}_{1}$ can predict a poor prognosis such as future deterioration. Therefore, periodic PFTs are essential for the optimal monitoring of COPD patients [2].

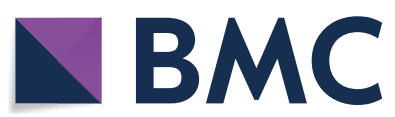

(c) The Author(s) 2021. Open Access This article is licensed under a Creative Commons Attribution 4.0 International License, which permits use, sharing, adaptation, distribution and reproduction in any medium or format, as long as you give appropriate credit to the original author(s) and the source, provide a link to the Creative Commons licence, and indicate if changes were made. The images or other third party material in this article are included in the article's Creative Commons licence, unless indicated otherwise in a credit line to the material. If material is not included in the article's Creative Commons licence and your intended use is not permitted by statutory regulation or exceeds the permitted use, you will need to obtain permission directly from the copyright holder. To view a copy of this licence, visit http://creativecommons.org/licenses/by/4.0/. The Creative Commons Public Domain Dedication waiver (http://creativeco mmons.org/publicdomain/zero/1.0/) applies to the data made available in this article, unless otherwise stated in a credit line to the data. 
The Korean Health Insurance Review and Assessment Service (HIRA) has conducted the national Asthma Quality Assessment Program since 2013 to improve the practical management and clinical outcomes of asthmatics [6], and the results of it showed that regular follow-up visits significantly reduced the risk for asthma exacerbation [7]. In 2014, HIRA initiated the National COPD Quality Assessment Program (CQAP) to minimize the national burden of COPD and achieve the optimal treatment strategy to improve the quality of medical services for COPD in Korea. The CQAP assessed several parameters in Korean medical institutions with the following protocol: (a) regular lung function monitoring, (b) regularly visiting, (c) inhaled bronchodilator prescription for COPD patients, (d) admission rate, and (e) emergency department visitation rate. Although the annual CQAP results have been released to the public, the direct and indirect impact of this program on clinical practice has not yet been estimated.

In this study, we investigated the influence of the CQAP on the treatment behavior of clinicians managing COPD, the clinical outcomes of COPD patients, and the national burden of COPD in Korea.

\section{Methods}

\section{The National COPD Quality Assessment Program}

The CQAP was proposed by the Korean government in 2014 and is conducted annually to (a) evaluate how COPD patients are treated in all Korean medical institutions; (b) improve the quality of COPD management protocols in clinics; and (c) improve the clinical outcomes and prognosis of COPD patients. It evaluates the following: (1) PFT conduction at least once during the 1-year assessment term; (2) regular follow-up visitation rate of COPD patients (i.e., at least three visits during the assessment term); and (3) inhaled bronchodilator prescription rate. The inhaled bronchodilator includes LAMA, long-acting $\beta 2$ agonists (LABA), short-acting $\beta_{2}$-agonists (SABA), a LABA + LAMA combination, and a $\mathrm{LABA}+$ inhaled corticosteroid combination drug.

After excluding institutions with $<10$ COPD patients, HIRA rated all medical institutions according to five grades (1-5, with a lower number indicating better COPD management) in accordance with the overall score of the CQAP results (100-80, 1st grade; 80-65, 2 nd grade; $50-65$, 3rd grade; $50-35$, 4 th grade; $<35,5$ th grade). The overall score was calculated by a weighted average of the PFT conduction rate $(40 \%)$, proportion of patients with regular follow-up (20\%), and inhaled bronchodilator prescription rate $(40 \%)$.

\section{Data extraction from claims data linked with the CQAP}

Korea has a unique national health insurance system covering all 50 million Korean people. Therefore, records of medical use, prescribed medications, and tests performed are included in the claims data. We extracted data from May 1, 2014 to April 30, 2017.

\section{Definition of COPD patients from HIRA claims data}

COPD patients were defined as those who visited a medical institution (outpatient and/or inpatient department) at least once during the assessment term to manage his/ her COPD. COPD patients who met the following criteria during the assessment term were assessed by the CQAP, and they were called as assessed subjects (AS): (1) age $\geq 40$ years; (2) ICD-10 codes for COPD (J43, emphysema and J44, other COPD) as the primary diagnosis or first sub-diagnosis; and (3) at least two separate outpatient visits with the prescription of COPD medications or at least one admission for treatment with systemic steroids followed by outpatient visits. In 2014, this case definition of AS (for COPD) was established by the HIRA committee according to the opinions of respiratory and critical care experts in Korea. Since then, this definition has been widely used by many researchers and data analysts $[8,9]$. COPD patients who did not meet the above criteria were not assessed by the CQAP, and they were classified as not-assessed subjects (NAS).

\section{Determination of clinical outcomes}

We defined the clinical outcomes as follows: (a) the number of admissions for treatment in a medical institution because of COPD exacerbation (with the ICD-10 codes of COPD [J43 and J44]); and (b) the yearly all-cause mortality rate.

\section{Study designs}

Figure 1a briefly depicts the study design and numbers of patients included in this study. We applied four different study models to analyze the CQAP results (Fig. 1b). First, we selected the AS in each year. Next, the NAS groups were created. The CQAP results of the six groups were evaluated and compared at each assessment term. Then, we calculated the risk reduction rate over a 3-year period in the AS group compared with that in the NAS group using the following equation: 1-(relative increase in risk in the AS over 3 years/relative increase in risk in the NAS over 3 years), with a higher positive value indicating positive risk reduction effects of the CQAP in the AS group compared with those in the NAS group. Second, we selected a subgroup of patients assessed by the CQAP for 3 consecutive years and compared their serial annual outcomes. Third, we performed a matching analysis to 


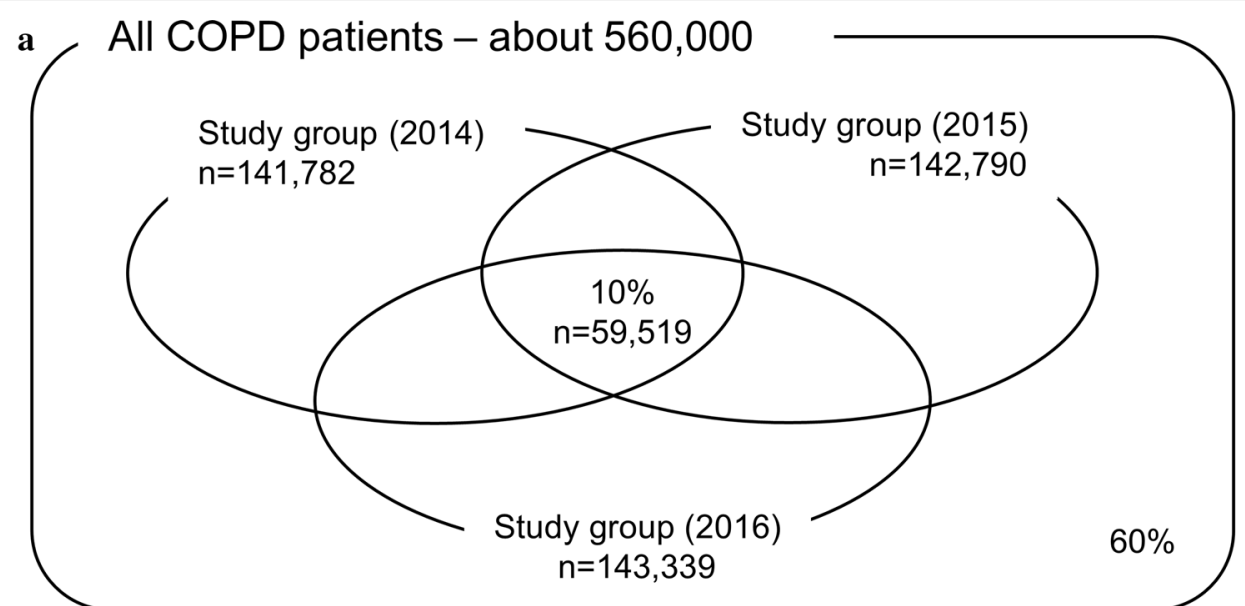

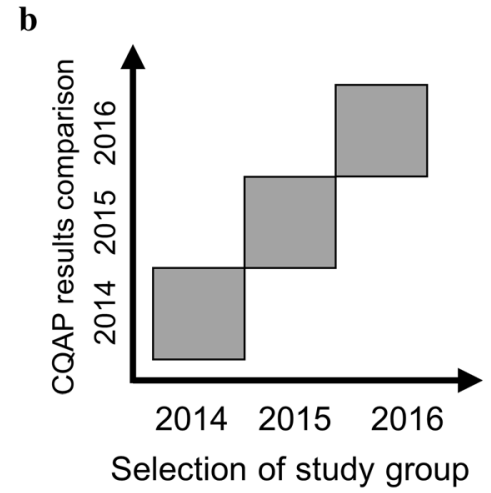

[Design 1: select subjects in each year \& assessment in each year]

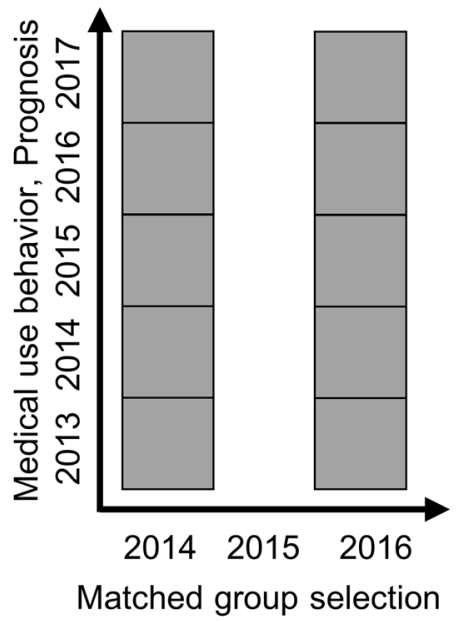

[Design 3: matching selection in 2014 \& 2016, assessment over 5 years]

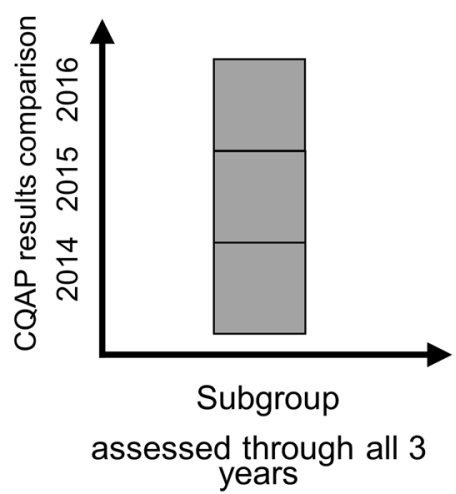

[Design 2: subgroup selection

$\&$ assessment over 3 years]

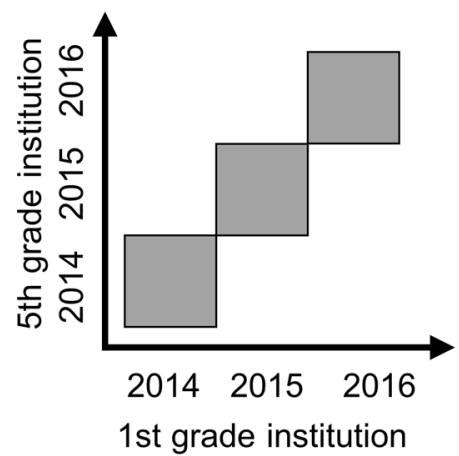

[Design 4: select institutions in each year $\&$ assessment in each year]

Fig. 1 Venn diagram for the COPD population included in this study (a) and conceptual diagrams showing four difference study designs (b). COPD, chronic obstructive pulmonary disease 
compare the sequential changes of the CQAP parameters and clinical outcomes of the same patients in 2014 and 2016. To minimize the confounding factors, age, sex, and type of medical institution visited were matched. Fourth, we compared the CQAP results and clinical outcomes of "1st grade" medical institutions (overall CQAP score: 100-80 points) versus "5th grade" institutions (overall CQAP score $<35$ points) in the AS and NAS groups, annually from 2014 to 2016 . Patients who did not visit any institution or who visited both "1st grade" and "5th grade" institutions were excluded in this design.

\section{Ethics requirements}

The Institutional Review Board of Severance Hospital (Number: 4-2018-0956) approved this study with a waiver for the acquisition of informed consent because of the minimal risk to patients.

\section{Statistical analyses}

We compared demographic data, medical use behaviors, and clinical outcomes between groups using the $\mathrm{X} 2$ test (for categorical variables), independent sample t-test, and analysis of variance test (for continuous variables). To estimate and compare the risk reduction rate over 3 years between the AS and NAS groups, we used a generalized linear model. Propensity score matching was used to reduce the imbalance of age, sex, and types of medical institutions. Data were analyzed using SAS Enterprise version 6.1 (SAS Institute Inc., Cary, NC, USA). A $P$-value $<0.05$ was considered to indicate statistical significance.

\section{Results}

Demographic data, clinical practice parameters, and prognosis of all COPD patients

A total of 569, 253 (2014), 557,682 (2015), and 539,708 (2016) COPD patients were recorded in the HIRA claims data. (Mean age: 67.5-68.2 years, Male: 60\%). PFT conduction rates and inhaled LAMA and LABA prescription rates increased steadily each year, while SABA prescription rates steadily decreased for all COPD patients overall (all $P$-values $<0.001)$. (Table 1 ).

\section{Study population}

Of the 560,000 COPD patients, approximately 142,000 (25.5\%) were annually assessed by the CQAP. Approximately $40 \%$ of all COPD patients were evaluated by the CQAP at least once over the 3 years. Moreover, $10 \%$ of all patients were assessed for all 3 consecutive years (Fig. 1a).

\section{Demographic data, clinical practice parameters, and prognosis in the AS and NAS groups}

In the AS groups, the PFT conduction rate increased from $60.2 \%$ to $69.0 \%$, the inhaled LAMA prescription rate increased from $45.1 \%$ to $51.8 \%$, and the inhaled LABA prescription rate increased from $49.4 \%$ to $53.4 \%$ (all $P<0.001$ ). In the NAS groups, the PFT conduction rates were significantly lower than those in the AS groups; however, the rate increased from $24.8 \%$ to $27.8 \%$ (all $P<0.001$ ). Similarly, the inhaled LAMA and LABA prescription rates in the NAS groups were also significantly lower than those in the AS groups (all $P<0.001$ ).

Table 1 Demographics, clinical practice parameters, and prognosis of all COPD patients

\begin{tabular}{llll}
\hline $\begin{array}{l}\text { Year } \\
\text { COPD patients in HIRA claim data }\end{array}$ & $\begin{array}{l}2014 \\
n=569,253\end{array}$ & $\begin{array}{l}2015 \\
n=557,682\end{array}$ & $\begin{array}{l}2016 \\
n=539,708\end{array}$ \\
\hline Demographics & & $67.5 \pm 13.2$ & $68.2 \pm 13.2$ \\
$\quad$ Age, mean \pm SD & $66.8 \pm 13.2$ & $341,160(61.2)$ & $328,170(60.8)$ \\
Sex, male (\%) & $349,505(61.4)$ & & $206,519(37.0)$ \\
Clinical practice parameters & & $90,871(16.3)$ & $208,952(38.7)$ \\
PFT conduction (\%) & $191,465(33.6)$ & $153,830(27.6)$ & $94,501(17.5)$ \\
Inhaled LAMA prescription (\%) & $78,044(13.7)$ & $295,227(52.9)$ & $148,101(27.4)$ \\
Inhaled LABA prescription (\%) & $144,130(25.3)$ & $69,349(12.4)$ & $283,804(52.6)$ \\
ICS prescription (\%) & $305,744(53.7)$ & & $67,717(12.6)$ \\
SABA prescription (\%) & $90,031(15.8)$ & $0.147 \pm 0.791$ & $0.162 \pm 0.869$ \\
Prognosis & & $375.7 \pm 1943.6$ & $423.2 \pm 2124.7$ \\
Frequency of admission for COPD /yr, mean \pm SD & $0.146 \pm 0.773$ & $21,114(3.8)$ & $23,417(4.3)$ \\
Medical cost for COPD management, mean \pm SD (USD) & $335.5 \pm 1665.2$ & $17,950(3.2)$ & \\
All-cause mortality in the next year (\%) & & & \\
\hline
\end{tabular}

SD, standard deviation; PFT, pulmonary function test; LAMA, long-acting muscarine antagonist; LABA, long-acting $\beta_{2}$ agonist; ICS, inhaled corticosteroid; SABA, shortacting $\beta_{2}$ agonist

Medical cost was calculated using exchange rate between dollar and Korean won (KRW) in Jan 1 st 2020 (1 dollar $=1167.5 \mathrm{KRW})$ 
In contrast, the admission frequencies for COPD and allcause mortality rates in the AS groups were higher than those in the NAS groups (Table 2).

\section{Comparison between the changes in CQAP parameters and risk reduction rate over 3 years}

PFT conduction rates and inhaled LAMA and LABA prescription rates increased over the 3 years for both groups. Significant changes in the clinical practice and prescription pattern for most COPD medications (except ICS) were observed in both groups. Furthermore, although the admission frequency because of COPD slightly decreased in the AS group over the 3 years $(-3.6 \%)$, it increased in the NAS group $(+22.0 \%)$ (risk reduction rate, $21.2 \%$; $P<0.001)$. Finally, the all-cause mortality rate greatly increased in the NAS group over the 3 years $(+65.2 \%)$, whereas it very slightly decreased in the AS group $(-0.3 \%)$ (risk reduction rate, $40.7 \% ; P<0.001$ ) (Table 3 ).

\section{Serial changes in clinical practice parameters} and prognosis in patients assessed by the CQAP for 3 consecutive years

Almost all of the above-mentioned CQAP variables related to clinical practice were high at baseline and remained constant over the assessment period in those evaluated by the CQAP over 3 consecutive years.
However, the ICS prescription rate decreased and the admission frequency significantly increased (Table 4).

\section{Matching analyses}

Matching analyses were conducted in the NAS-2016 and AS-2016 groups $(n=127,927$, respectively) and NAS2014 and AS-2014 groups $(n=137,402$, respectively). Although the PFT conduction rate and inhaled LAMA prescription rate in matched AS-2016 patients peaked in 2016, they decreased to the level of them in 2017. However, the admission frequency in the matched AS-2016 patients increased in 2016 and continued increasing in 2017. In contrast, the above values were not greatly influenced by the assessment term in the matched NAS-2016 group (Fig. $2 \mathrm{a}-\mathrm{c}$ ). Similar results were observed in the matching analyses between the NAS-2014 and AS-2014 groups (Figs. 2d-f).

\section{Comparison of clinical practice parameters with the prognosis of COPD patients according} to the grade of the medical institution regularly visited In the AS groups, COPD patients who visited the "1st grade" medical institutions reported higher PFT conduction rates and an improvement of this rate $(81.6 \%$ to $84.0 \%)$ than those who regularly visited the " 5 th grade" institutions (3.4\% to $3.3 \%)$. Rates of inhaled LAMA

Table 2 Demographics, clinical practice parameters and prognosis of study groups

\begin{tabular}{|c|c|c|c|c|c|c|}
\hline \multirow{2}{*}{$\begin{array}{l}\text { Year } \\
\text { Study group } \\
\text { (number) }\end{array}$} & \multicolumn{2}{|l|}{2014} & \multicolumn{2}{|l|}{2015} & \multicolumn{2}{|l|}{2016} \\
\hline & $\begin{array}{l}\text { AS-2014 } \\
(n=141,782)\end{array}$ & $\begin{array}{l}\text { NAS-2014 } \\
(n=427,471)\end{array}$ & $\begin{array}{l}\text { AS-2015 } \\
(n=142,790)\end{array}$ & $\begin{array}{l}\text { NAS-2015 } \\
(n=414,892)\end{array}$ & $\begin{array}{l}\text { AS-2016 } \\
(n=143,339)\end{array}$ & $\begin{array}{l}\text { NAS-2016 } \\
(n=396,369)\end{array}$ \\
\hline \multicolumn{7}{|l|}{ Demographics } \\
\hline Age, mean $\pm S D$ & $69.9 \pm 10.1$ & $65.8 \pm 13.9$ & $70.0 \pm 10.1$ & $66.7 \pm 14.0$ & $70.1 \pm 10.1$ & $67.5 \pm 14.1$ \\
\hline Sex, male (\%) & $101,795(71.8)$ & $247,710(57.9)$ & $103,882(72.8)$ & $237,278(57.2)$ & $106,664(74.4)$ & $221,506(55.9)$ \\
\hline \multicolumn{7}{|c|}{ Clinical practice parameters } \\
\hline PFT conduction (\%) & $85,392(60.2)$ & $106,073(24.8)$ & $91,084(63.8)$ & $115,435(27.8)$ & $98,895(69.0)$ & $110,957(27.8)$ \\
\hline $\begin{array}{l}\text { Inhaled LAMA } \\
\text { prescription (\%) }\end{array}$ & $63,932(45.1)$ & $14,112(3.3)$ & $69,833(48.9)$ & $21,038(5.1)$ & $74,254(51.8)$ & $20,247(5.1)$ \\
\hline $\begin{array}{l}\text { Inhaled LABA pre- } \\
\text { scription (\%) }\end{array}$ & 70,091 (49.4) & $74,039(17.3)$ & 76,597 (53.6) & $77,233(18.6)$ & $76,508(53.4)$ & $71,593(18.1)$ \\
\hline ICS prescription (\%) & $82,331(58.1)$ & $223,413(52.3)$ & $81,284(56.9)$ & $213,943(51.6)$ & $81,242(56.7)$ & $202,562(51.1)$ \\
\hline $\begin{array}{l}\text { SABA prescription } \\
(\%)\end{array}$ & $51,066(36.0)$ & $38,965(9.1)$ & $30,158(21.1)$ & $39,191(9.5)$ & 29,931 (20.9) & $37,786(9.5)$ \\
\hline \multicolumn{7}{|l|}{ Prognosis } \\
\hline $\begin{array}{l}\text { Frequency of } \\
\text { admission } \\
\text { for COPD/yr, } \\
\text { mean } \pm S D\end{array}$ & $0.307 \pm 1.062$ & $0.091 \pm 0.634$ & $0.281 \pm 1.002$ & $0.100 \pm 0.695$ & $0.296 \pm 1.065$ & $0.111 \pm 0.777$ \\
\hline $\begin{array}{l}\text { All-cause mortality } \\
\text { in the next year } \\
(\%)\end{array}$ & $8278(5.8)$ & $9672(2.3)$ & $8217(5.8)$ & $12,897(3.1)$ & $8341(5.8)$ & $15,076(3.8)$ \\
\hline
\end{tabular}

AS, assessed subjects; NAS, not-assessed subjects; SD, standard deviation; PFT, pulmonary function test; LAMA, long-acting muscarine antagonist; LABA, long-acting $\beta_{2}$ agonist; ICS, inhaled corticosteroid; SABA, short-acting $\beta_{2}$ agonist 
Table 3 Comparison between the changes in CQAP parameters and risk reduction rate over 3 years

\begin{tabular}{|c|c|c|c|c|}
\hline & AS & NAS & $\begin{array}{l}\text { Risk reduction rate in AS compared } \\
\text { to NAS }\end{array}$ & $P$-value \\
\hline \multicolumn{5}{|l|}{ Clinical practice parameters } \\
\hline PFT conduction rate & $+14.6 \%$ & $+12.1 \%$ & $-2.4 \%$ & $<0.001$ \\
\hline Inhaled LAMA prescription rate & $+14.9 \%$ & $+54.5 \%$ & $+25.8 \%$ & $<0.001$ \\
\hline Inhaled LABA prescription rate & $+8.1 \%$ & $+4.6 \%$ & $-3.5 \%$ & $<0.001$ \\
\hline ICS prescription rate & $-2.4 \%$ & $-2.2 \%$ & $+0.2 \%$ & $<0.001$ \\
\hline SABA prescription rate & $-41.9 \%$ & $+4.4 \%$ & $+44.6 \%$ & $<0.001$ \\
\hline \multicolumn{5}{|l|}{ Prognosis } \\
\hline Frequency of admission for COPD & $-3.6 \%$ & $+22.0 \%$ & $+21.2 \%$ & $<0.001$ \\
\hline All-cause mortality in the next year & $-0.3 \%$ & $+65.2 \%$ & $+40.7 \%$ & $<0.001$ \\
\hline
\end{tabular}

AS, assessed subjects; NAS, not-assessed subjects; PFT, pulmonary function test; LAMA, long-acting muscarine antagonist; LABA, long-acting $\beta_{2}$ agonist; ICS, inhaled corticosteroid; SABA, short-acting $\beta_{2}$ agonist

Table 4 Serial changes of clinical practice parameters and prognosis in subjects assessed by CQAP for consecutive 3 years

\begin{tabular}{|c|c|c|c|c|}
\hline $\begin{array}{l}\text { Year } \\
\text { Overlapping patients in AS group for } 3 \text { years }\end{array}$ & $\begin{array}{l}2014 \\
n=59,519\end{array}$ & $\begin{array}{l}2015 \\
n=59,519\end{array}$ & $\begin{array}{l}2016 \\
n=59,519\end{array}$ & $\begin{array}{l}\text { 3-year changes } \\
(\%)\end{array}$ \\
\hline \multicolumn{5}{|l|}{ Clinical practice parameters } \\
\hline PFT conduction (\%) & $40,258(67.6)$ & $39,390(66.2)$ & $40,831(68.6)$ & +1.5 \\
\hline Inhaled LAMA prescription (\%) & $36,700(61.7)$ & $37,851(63.6)$ & $36,907(62.0)$ & +0.5 \\
\hline Inhaled LABA prescription (\%) & $35,746(60.1)$ & $37,800(63.5)$ & $36,656(61.6)$ & +2.5 \\
\hline ICS prescription (\%) & $33,468(56.2)$ & $32,118(54.0)$ & $32,231(54.2)$ & -3.6 \\
\hline SABA prescription (\%) & $15,029(25.3)$ & $14,248(23.9)$ & $14,516(24.4)$ & -3.6 \\
\hline \multicolumn{5}{|l|}{ Prognosis } \\
\hline Frequency of admission for COPD/yr, mean $\pm S D$ & $0.288 \pm 0.923$ & $0.277 \pm 0.969$ & $0.372 \pm 1.260$ & +29.2 \\
\hline
\end{tabular}

AS, assessed subjects; PFT, pulmonary function test; LAMA, long-acting muscarine antagonist; LABA, long-acting $\beta_{2}$ agonist; ICS, inhaled corticosteroid; SABA, shortacting $\beta_{2}$ agonist

prescription were also higher among the former patients than among the latter patients. However, the mean admission frequency because of COPD was higher among patients who visited "1st grade" institutions than among the patients who visited "5th grade" institutions $(P<0.001)$. The all-cause mortality rate was higher among the former patients than among the latter patients $(P<0.001)$. The mean risk ratio of mortality to admission frequency for the 3 years was about four times higher among the former patients than among the latter patients (Fig. 3).

\section{Discussion}

The CQAP conducted by HIRA in Korea is a government-driven specific disease-oriented management qualifying program. It aims to assess the level of management protocols achieved for COPD patients in all medical institutions in Korea. HIRA publishes the results of the CQAP annually with information regarding the COPD management quality of each medical institution and ratings from "grade 1" to "grade 5" [10]. In this study, we revealed that the Korean CQAP positively influenced the quality of COPD medical services. Since the start of this program in 2014, the PFT conduction and inhaled bronchodilator prescription rates have steadily increased. Furthermore, these improvements were greater in the AS group than in the NAS group. It can be assumed that the CQAP has some influence on clinicians' practices and patients' medical use behavior in real-world settings.

The CQAP also influenced COPD management and prognosis. This phenomenon appeared differently in the AS and NAS groups. Every year, the CQAP data showed that the percentage of prescribed bronchodilators in the AS group was higher than that in the NAS group. Every year, the it also showed that the admission rate of patients in the AS group because of COPD was higher than that of patients in the NAS group; the mortality rate was also higher in the AS group. It is thought that patients with more severe COPD were included in the AS group than in the NAS group. We speculate that this phenomenon is because the coverage of medical insurance increased with national income and the interest in the health of 


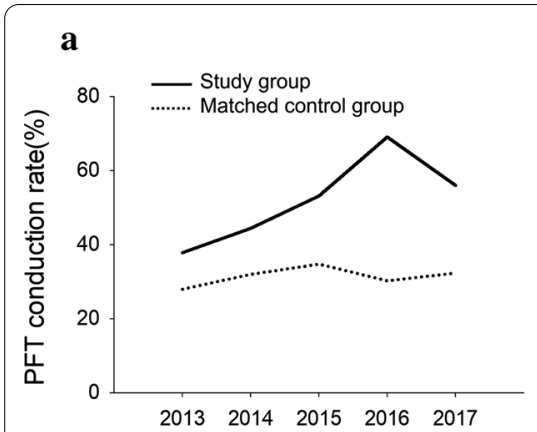

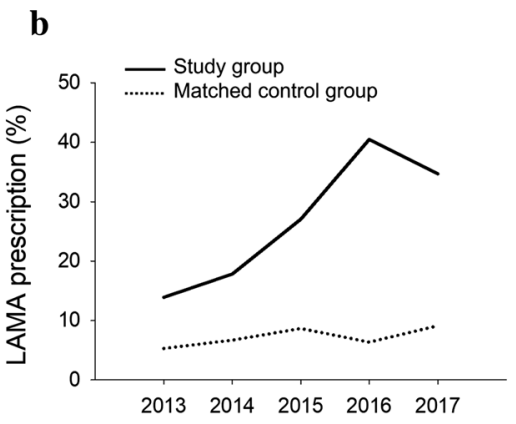

e

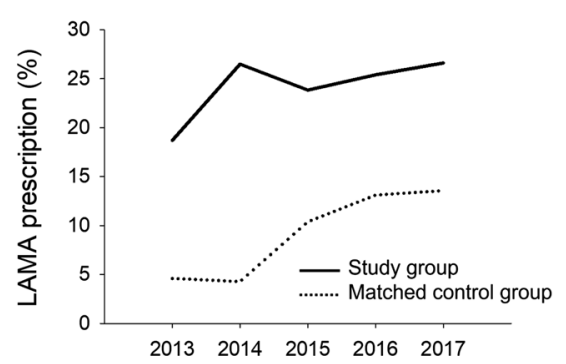

c

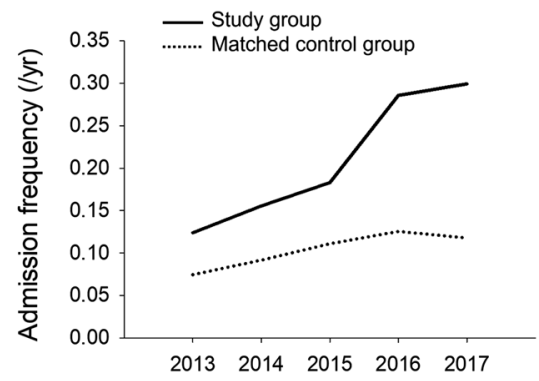

f

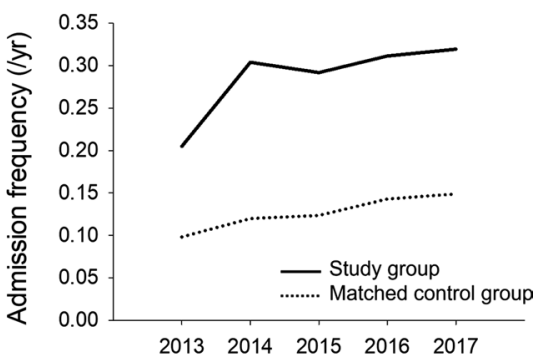

Fig. 2 Matching analyses. Comparison of annual changes in the PFT conduction rate (a), inhaled LAMA prescription rate (b), and admission frequency $(\mathbf{c})$ between those patients assessed by CQAP and matched patients that were not assessed in 2016. Comparison of annual changes in the PFT conduction rate (a), inhaled LAMA prescription rate (b), and admission frequency (c) between patients assessed by CQAP and matched patients that were not assessed in 2014. PFT, pulmonary function test; COPD, chronic obstructive pulmonary disease. CQAP, COPD quality assessment program

chronically ill patients increased. Additionally, because of the natural course of COPD, the mortality rate of severely ill patients is inevitably high.

However, when analyzing the results for 3 years in a row, the hospitalization and mortality rates in the AS group slightly decreased, while in the NAS group both rates increased significantly. The risk reduction rates of the AS group for this result were $21.2 \%$ and $40.7 \%$, and thorough COPD management through the CQAP played a major role. The guidelines suggest that the use of inhaled bronchodilators has a positive effect on the management and prognosis of COPD patients, but they are not widely used in practice in Korea. This implies that both clinicians and patients need to change their perceptions about long-term management goals of COPD [11]. Additionally, it is well known that periodic monitoring using PFTs can predict a patient's prognosis, but it is doubtful whether they are performed in real practice situations [12].

To reduce the socioeconomic burden of chronic illnesses, the physicians managing chronic illnesses should manage patients in the most efficient way possible (with regards to factors such as guidelines and action plans), and patients should show a high degree of adherence to the control and rescue medications [13, 14]. The CQAP is considered a new model for inducing changes in the behavior of doctors and patients. For asthma, HIRA has been conducting the Asthma Quality Assessment Program (AQAP) since 2013. A 4-year AQAP analysis showed that the risk reduction rates of admission frequency because of asthma exacerbation and all-cause mortality rate of the patients observed by AQAP (AS group) were $17.1 \%$ and $24.4 \%$. when compared with not assessed subjects (NAS group) [15]. These were lower than those of COPD because the decline in lung function and irreversible changes are more severe in COPD than in asthma, and the probability of systemic co-morbidity is higher in COPD.

However, weak effects were observed in the subgroup assessed by the CQAP for 3 consecutive years. This subgroup had higher PFT conduction and prescription rates of LABA and LABA inhalers at baseline. A higher admission frequency was found compared to those in the NAS group. This subgroup required multiple COPD medications and was highly dependent on high-quality (upper rather than secondary hospital) medical services, implying that this subgroup experienced more severe COPD. Unfortunately, this group did not much improvement.

Additionally, the positive effects of the CQAP may be temporary. In the matching analysis, the PFT conduction 

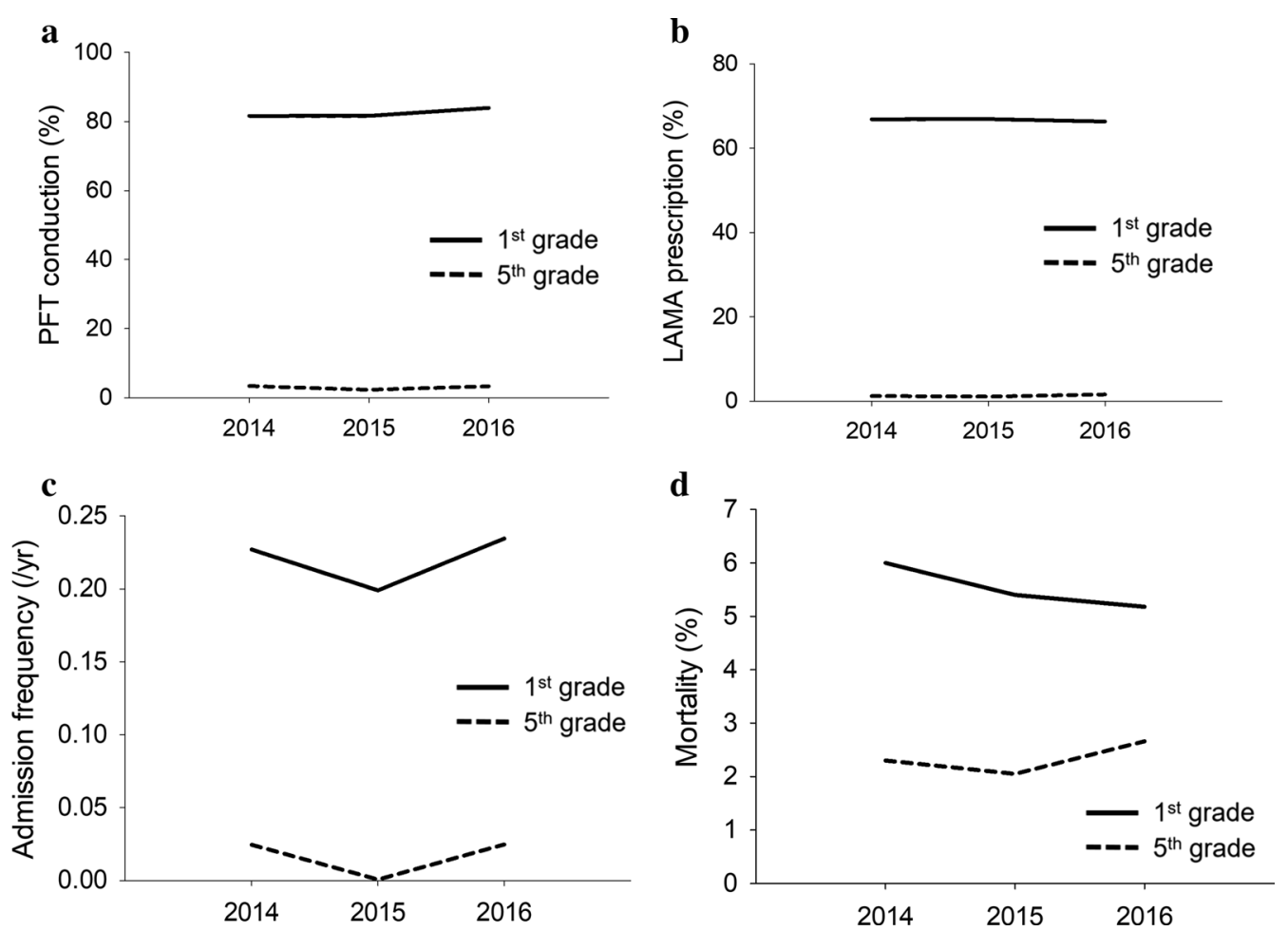

Fig. 3 Comparison of annual changes of PFT conduction rate (a), inhaled LAMA prescription rate (b), and admission frequency (c) and all-cause mortality (d) between CQAP enrolled patients who visited the "1st grade" medical institution and those who visited the "5th grade" institution. PFT, pulmonary function test; COPD, chronic obstructive pulmonary disease. CQAP, COPD quality assessment program, LAMA, long-acting muscarine antagonists

and LAMA prescription rates of the AS-2016 group showed a sharp decrease in 2017. Such a phenomenon was also observed in the AS-2014 group. This means that government-led policies alone have limits to the duration of effects on the positive behavior changes of physicians. Therefore, in continuously operating programs such as the CQAP, through the close cooperation with academia as a private sector, doctors themselves can autonomously modify their practices for improvement.

HIRA classify all medical institutions in Korea into five grades considering management protocol of COPD, and made this information publicly available. We can expect 1st grade institutions show better practice performance the compared to the 5th grade institutions, however, the hospitalization and mortality rates increased as the grade of the institution decreased (5th to 1st grade). This can be explained by considering the size of the medical institutions and the severity of COPD. Overall, Korean COPD patients are more likely to be treated by higher-level institutions for more severe illnesses because of continuous pulmonary dysfunction, and are monitored and managed according to global guidelines. Therefore, how CQAP guides mild-to-moderate patients to be treated and monitored according to guidelines at 3rd-, 4th-, and 5th-grade institutions is the main question to be focused in the future.

Although it seems that the prognosis of patients who visit 1st grade institutions is poorer than that of patients who visit 5th grade institutions, we confirmed that improvement of PFT conduction is better in the former. In addition, while admission frequency in the former is about 10 times higher than in the latter, the mortality rate in 1st grade institutions is only about 2.5 times higher. This implies that the mortality risk of patients admitted to 1st grade institutions is only about one quarter of that of those admitted to 5th grade institutions. We found that institutions highly rated by HIRA showed a better prognosis than those rated lower. Therefore, we need to encourage clinicians to improve COPD management protocols as suggested by HIRA to improve their rating because it will help improve the prognosis of COPD patients.

The NAS group was younger and consisted of more females compared to the AS group. The NAS group might include mild COPD patients who did not need COPD medication regularly. Because aging and smoking history are critical factors for development and progression of COPD, young female patients might have relatively mild 
COPD. Additionally, female COPD patients might be ashamed and afraid to be diagnosed with COPD. Then, they may not visit the hospital or be frequently medicated for COPD. We speculated these reasons might lead to NAS group to be predominantly consisting of young females.

The percentage of prescribed ICS in the AS and NAS groups was similar, whereas percentage of prescribed bronchodilator in the AS group is higher than that in the NAS group. This could mean that the NAS group did not have proper management (including bronchodilator), and they used ICS or oral medication to relieve their symptoms. Additionally, we did not exclude asthma patients to clarify real-world characteristics of COPD. Consequently, these might lead to a high percentage of prescribed ICS.

This study has strengths from several perspectives. First, this is the first study to reveal a positive influence of the national health insurance program and the CQAP, and an improvement in the overall prognosis of COPD using four different study designs. Second, we assessed the complete national insurance cohort HIRA database to reduce selection bias. Third, it reveals the multi-faceted positive influences of the CQAP using a variety of study designs.

This study has some limitations. First, COPD patients were defined with diagnostic codes only. Second, this study was not designed with randomization; thus, the direct effects of the CQAP could not be ascertained. Third, the study results should not be interpreted broadly for application in countries that do not implement a national medical insurance system. Fourth, there is the possibility of an unmeasured negative impact of the CQAP (example: the intentional removal or insertion of diagnostic codes, over-prescription of COPD medications, and avoidance of non-adherent patients). Last, we did not define COPD exacerbation by medication use including antibiotics or steroids.

\section{Conclusions}

We found a positive influence of the CQAP conducted by government on both the patient management protocol and prognosis of COPD in Korea. The CQAP was found to be effective overall, but restricted to the annual assessment term. Thus, we believe that the continued application of this type of quality assessment program will help improve clinical outcomes and medical utilization for COPD patients.

\footnotetext{
Abbreviations

AS: Assessed subjects; Cl: Confidence interval; COPD: Chronic obstructive pulmonary disease; CQAP: COPD quality assessment program; GOLD: Global Initiative for Chronic Obstructive Lung Disease; HIRA: Health Insurance Review and Assessment Service; ICS: Inhaled corticosteroids; LABA: Long-acting $\beta_{2}$ agonists; LAMA: Long-acting muscarine antagonists; NAS: Not-assessed
}

subjects; OR: Odds ratio; PFT: Pulmonary function test; SABA: Short-acting $\beta_{2}$-agonists.

\section{Acknowledgements}

We really appreciate to have opportunity to participate in Joint Project on Quality Assessment Research in 2019.

\section{Authors' contributions}

HJP contributed to the ideation and design of this study; analyzed and interpreted the data; drafted and revised the article; and approved the final version of this manuscript for publication. SRK, BYK, HKK, SIA, and JHS collected and analyzed the data; contributed to the preparation of the draft; revised the article; and approved the final version of this manuscript for publication. SK and HSL are professional biostatisticians and are responsible for the analysis and interpretation of the data. JHL provided constructive criticism on the rationale and design of this study; interpreted the data; drafted and revised the article; and approved the final version of this manuscript for publication. As the corresponding author, he guarantees the paper and takes responsibility for the integrity of the work as a whole. Finally, JWP helped with the ideation of this study; designed the study; and greatly contributed to the draft and final version of this manuscript. All authors read and approved the final manuscript.

\section{Funding}

This research did not receive any specific grant from funding agencies in the public, commercial, or not-for-profit sectors.

\section{Availability of data and materials}

The datasets used and analysed during the current study are available from the corresponding author on reasonable request.

\section{Declarations}

\section{Ethic approval and consent to participate}

The Institutional Review Board of Severance Hospital (Number: 4-2018-0956) approved this study with a waiver for the acquisition of informed consent because of the minimal risk to patients.

\section{Consent for publication}

Not applicable.

\section{Competing interests}

The authors declare that they have no competing interests.

\section{Author details}

${ }^{1}$ Department of Internal Medicine, Gangnam Severance Hospital, Yonsei University College of Medicine, Seoul, Republic of Korea. ${ }^{2}$ Division of Allergy and Immunology, Department of Internal Medicine, Yonsei University College of Medicine, Seoul, Republic of Korea. ${ }^{3}$ Institute of Allergy, Yonsei University College of Medicine, Seoul, Republic of Korea. ${ }^{4}$ Division of Biostatistics, Department of R\&D Management, Kangbuk Samsung Hospital, Sungkyunkwan University School of Medicine, Seoul, Republic of Korea. ${ }^{5}$ Biostatistics Collaboration Unit, Yonsei University College of Medicine, Seoul, Republic of Korea. ${ }^{6}$ Healthcare Insurance Review and Assessment Service, Wonju, Republic of Korea.

Received: 25 November 2020 Accepted: 14 March 2021

Published online: 21 March 2021

\section{References}

1. Agusti A, Hogg JC. Update on the pathogenesis of chronic obstructive pulmonary disease. N Engl J Med. 2019;381:1248-56.

2. Chen S, Wang C, Li B, Shi G, Li H, Zhang J, Gu Y, Zhou J, Song Y, Bai C. Risk factors for FEV1 decline in mild COPD and high-risk populations. Int J Chron Obstruct Pulmon Dis. 2017:12:435-42.

3. Celli BR, Wedzicha JA. Update on clinical aspects of chronic obstructive pulmonary disease. N Engl J Med. 2019;381:1257-66.

4. Collaborators GCD: GBD. Causes of Death Collaborators. Global, regional, and national age-sex-specific mortality for 282 causes of death in; 195 
countries and territories, 1980-2017: a systematic analysis for the Global Burden of Disease Study 2017. Lancet. 2017;2018(392):2170-2170.

5. Rabe KF, Watz H. Chronic obstructive pulmonary disease. Lancet. 2017;389:1931-40.

6. Choi JY, Yoon HK, Lee JH, Yoo KH, Kim BY, Bae HW, Kim YK, Rhee CK. Current status of asthma care in South Korea: nationwide the Health Insurance Review and Assessment Service database. J Thoracic Dis. 2017;9:3208-14

7. Park HJ, Byun MK, Kim HJ, Ahn CM, Rhee CK, Kim K, Kim BY, Bae HW, Yoo $\mathrm{KH}$. Regular follow-up visits reduce the risk for asthma exacerbation requiring admission in Korean adults with asthma. Allergy Asthma Clin Immunol. 2018;14:29.

8. Lee J, Lee JH, Kim JA, Rhee CK. Trend of cost and utilization of COPD medication in Korea. Int J Chron Obstruct Pulmon Dis. 2017;12:27-33.

9. Chung SM, Lee SY. Evaluation of appropriate management of chronic obstructive pulmonary disease in Korea: based on health insurance review and assessment service (HIRA) claims. Tuberc Respir Dis (Seoul). 2017:80:241-6.

10. The HIRA et al. The report of asthma quality assessment program. Korea: Commitee on Government. http://www.hira.or.kr/re/diag/getDiagEvlList. do?pgmid=HIRAA030004000100. Accessed 13 Aug 2020.

11. Di Martino M, Agabiti N, Bauleo L, Kirchmayer U, Cascini S, Pistelli R, Colamesta V, Patorno E, Pinnarelli L, Fusco D, et al. Use patterns of long-acting bronchodilators in routine COPD care: the OUTPUL study. Copd. 2014;11:414-23.

12. Singh D, Criner GJ, Naya I, Jones PW, Tombs L, Lipson DA, Han MLK. Measuring disease activity in COPD: is clinically important deterioration the answer? Resp Res. 2020. https://doi.org/10.1186/s12931-020-01387-z.

13. Gaude GS, Hattiholi J, Chaudhury A. Role of health education and selfaction plan in improving the drug compliance in bronchial asthma. J Family Med Prim Care. 2014;3:33-8.

14. Laba TL, Bleasel J, Brien JA, Cass A, Howard K, Peiris D, Redfern J, Salam A Usherwood T, Jan S. Strategies to improve adherence to medications for cardiovascular diseases in socioeconomically disadvantaged populations: a systematic review. Int J Cardiol. 2013;167:2430-40.

15. Park HJ, Kim SR, Kim S, Lee HS, Kim BY, Kim HK, Ahn SI, Shin JH, Lee JH, Park JW. Impact of the asthma quality assessment program on burden of asthma. J Allergy Clin Immunol Pract. 2021;1:419-25.

\section{Publisher's Note}

Springer Nature remains neutral with regard to jurisdictional claims in published maps and institutional affiliations.
Ready to submit your research? Choose BMC and benefit from:

- fast, convenient online submission

- thorough peer review by experienced researchers in your field

- rapid publication on acceptance

- support for research data, including large and complex data types

- gold Open Access which fosters wider collaboration and increased citations

- maximum visibility for your research: over $100 \mathrm{M}$ website views per year

At BMC, research is always in progress.

Learn more biomedcentral.com/submissions 